\title{
Multilobulated popliteal cyst after a failed total knee arthroplasty
}

\author{
Biagio Moretti $\cdot$ Vittorio Patella $\cdot$ Elyazid Mouhsine $\cdot$ \\ Vito Pesce $\cdot$ Antonio Spinarelli $\cdot$ Raffaele Garofalo
}

Received: 16 June 2005/ Accepted: 12 April 2006/ Published online: 5 August 2006

(C) Springer-Verlag 2006

\begin{abstract}
Popliteal cyst is a rare finding after total knee arthroplasty (TKA), but when present, it might indicate a malfunction of the TKA related to generation of wear-particles, or loosening. We present a case of a multilobulated popliteal cyst developing in a patient 8 years after primary TKA. The cyst was associated with a mechanical prosthetic loosening. The primary complaint of the patient was pain in the posterior region of the knee. A two-stage procedure consisting of cyst excision at first, followed after 5 months by a revision TKA was performed. Intraoperatively, a darkish, multilobulated cyst with a well-defined thick wall filled with fluid containing polyethylene debris, communicating with the knee joint was found. After 3 years of follow-up, the patient was satisfied and walked without the support of a cane. The patient presented a satisfactory knee range of motion. Clinical, radiological and ultrasound investigations ruled out popliteal cyst recurrence. A dissecting popliteal cyst associated with a failed TKA should be excised because it contains polyethylene debris that constitutes an induced factor for prosthetic loosening. A two-stage procedure with quite a long time in-between, as
\end{abstract}

B. Moretti $\cdot$ V. Patella $\cdot$ V. Pesce $\cdot$ A. Spinarelli

Department of Clinical Methodology

and Surgical Technique, Orthopedics Section II,

University of Bari, Bari, Italy

E. Mouhsine $(\varangle) \cdot$ R. Garofalo

Department of Traumatology and Orthopaedic Surgery,

University Hospital, OTR-BH 14,

CHUV 1011 Lausanne, Switzerland

e-mail: Elyazid.Mouhsine@hospvd.ch presented in this paper, can be a useful alternative to manage such a problem, in particular in very old patients associated with other medical problems.

Keywords Total knee arthroplasty $\cdot$ Synovial cyst . Polyethylene wear $\cdot$ Revision $\cdot$ Loosening

\section{Introduction}

The popliteal cyst, first reported by Adams [1] originates from a distension of semimembranous bursa, as a result of different intraarticular knee pathologies. In the recent years, a dissecting popliteal cyst has been reported in cases of a malfunction in total knee arthroplasty (TKA) $[4,5,7,11,12]$. Despite prosthetic design and biomaterial improvement, the wear of a TKA still remains an uneventful event which may cause periprosthetic osteolysis, recidivant synovitis and foreign-body reactions affecting the longevity of a TKA. Patients with popliteal cysts usually complain of calf symptoms, such as sural pain during activity, limp, leg edema and a positive Homan's sign: in fact, Katz [9] called it "pseudotrombophlebitis". The presence of popliteal cyst in patients who previously underwent a TKA may be an indirect sign of prosthetic component's wear or loosening $[4,5,7,11,12]$. The popliteal cyst in this condition usually contains fluid filled with polyethylene debris $[5,12]$. The therapeutical approach to this problem is variable and debated among orthopaedic surgeons. In some reports $[5,12]$, the patients were managed by component revision, but excision of the popliteal cyst was not performed because of symptoms without signs of deterioration. Other authors [11] have suggested a single-stage procedure 
consisting in changing the worn-out prosthetic components combined with cyst excision. Another suggested strategy was a two-stage procedure consisting of cyst excision at first and then revision of the TKA in a second procedure. This two-stage procedure was based on patient's referred symptoms.

The purpose of this paper is to describe a clinical case of popliteal cyst that occurred after a failed TKA. The case was managed using a two-stage procedure; first the cyst was excised to reduce the pain, and after 5 months, the worn-out TKA was surgically revised. Moreover, the authors discuss the management and diagnosis of this clinical problem, and try to elucidate the origin and relationship between the cyst and the prosthetic implants.

\section{Case report}

A 72-year-old woman with primary knee osteoarthritis underwent right TKA (Genesis I, Smith-Nephew Inc, Memphis, TN, USA). She recovered with no complication. Her postoperative course as well as her radiographic controls were uneventful for 8 years. At that time, the patient noted the development of a progressive posterior joint effusion, associated with rest and exertional pain, as well as limp and crepitus during knee motion. Physical examination revealed a mild lower leg swelling, the presence of a firm, large and painful lobulated mass in the posteromedial aspect of the knee joint, and extending into the calf. The active range of motion of the operated knee was $40^{\circ}-5^{\circ}$ and a loud crepitation was audible. Homan's sign was positive. The peripheral vascular status was normal. Laboratory data showed a normal blood count, erythrocyte. Sedimentation rate and C-reactive protein results were of normal range. The coagulation parameters (prothrombin time, activate partial thromboplastin time, fibrinogen and D-dimers) were normal. Standard A-P X-rays of the knee revealed a wear to the medial compartment of the prosthesis, and a large soft tissue shadow in the popliteal area (Fig. 1). Doppler examination ruled out a deep venous thrombosis, and showed a cyst containing fluid, located in the popliteal region. CT-scan (Fig. 2) revealed a cyst averaging $8 \mathrm{~cm}$ of diameter filled with a "fine corpuscoled" material.

The decision of a surgical management was taken, and because the principal complaint of the patient was the pain, an excision of the cyst was first carried out, using a posterior approach. A voluminous cyst $\left(20 \times 11 \times 8 \mathrm{~cm}^{3}\right)$ was found in the popliteal area. The cyst appeared dark, multilobulated, with a well-defined

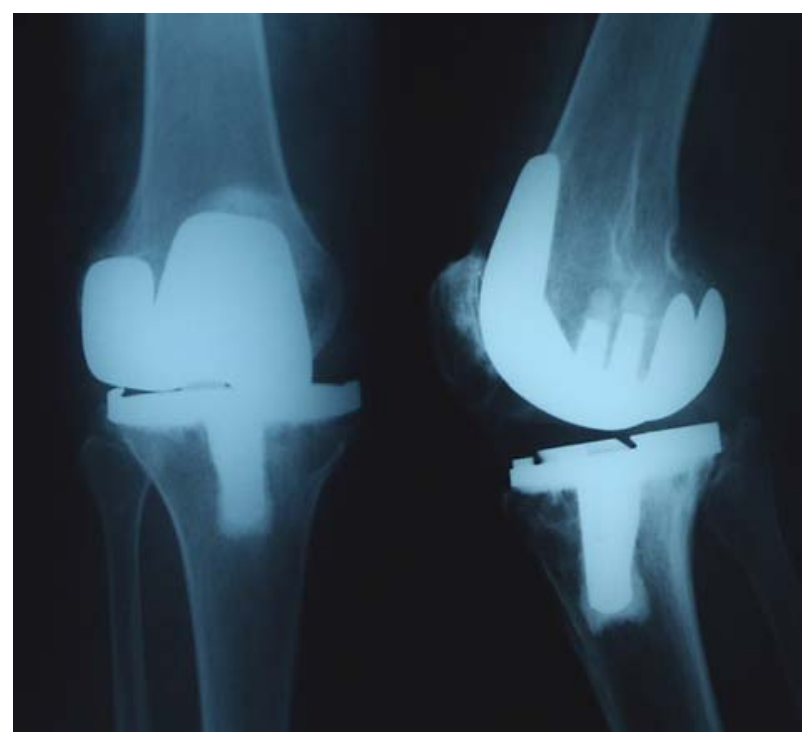

Fig. 1 Preoperative X-ray of the knee showing a large soft tissue shadow and an important usure of polyethylene in particular on medial side

thick cystic wall (Fig. 3) filled with fluid containing polyethylene debris, communicating with the knee joint. Polarized microscopic examination showed fibrous tissue with metallosis in the cyst wall, a foreignbody inflammatory reaction with presence of giant cells around crystals of polyethylene debris (Fig. 4). During the immediate postoperative period the patient was painless. After 5 months a revision surgery using a hinged prosthesis (Butterfly, Permedica SPA Merate, Italy) was carried out. Intraoperatively, prominent

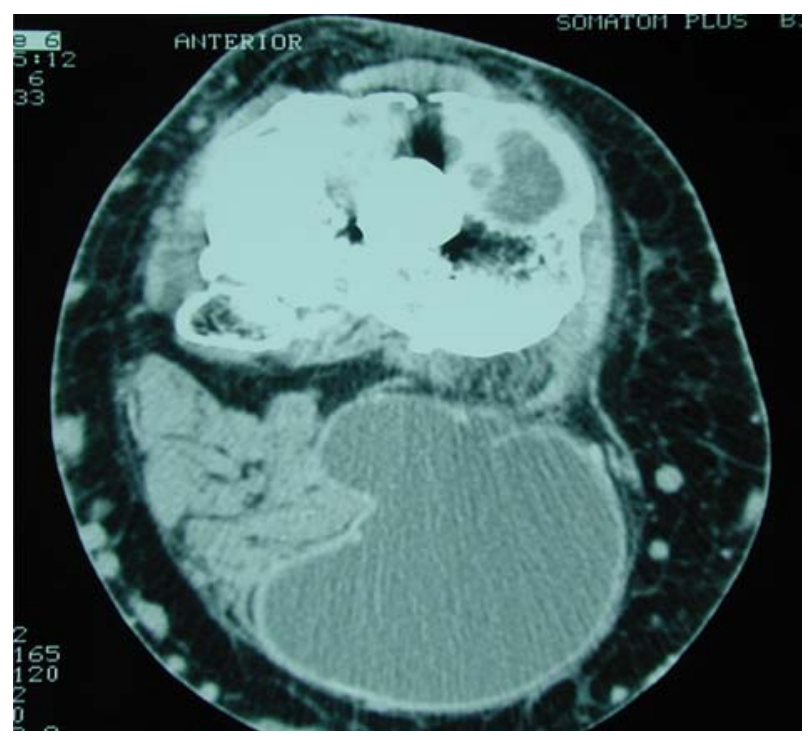

Fig. 2 Preoperatively CT-scan revealed a cystic mass of average $8 \mathrm{~cm}$ of diameter extending in the posteromedial region of the knee 


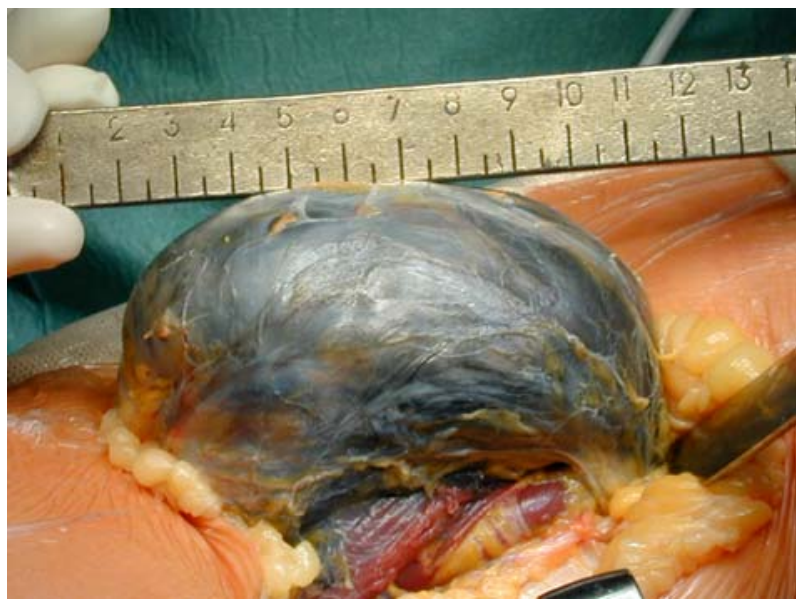

Fig. 3 The intraoperatively aspect of the voluminous popliteal cyst with a dark aspect

villous synovial proliferation with a blackish stain and a metal particle deposit was observed. Synoviectomy was performed. The retrieved polyethylene insert showed delamination, and the tibial implant was worn out on the medial side (Fig. 5). The postoperative course was uneventful. At 3 years of follow-up, the patient was satisfied; she walked with no cane, and the knee range of motion was $100^{\circ}-0^{\circ}$. Clinical, radiological and ultrasound investigations ruled out popliteal cyst recurrence (Fig. 6).

\section{Discussion}

In this paper we report the case of a patient who developed a dissecting popliteal cyst 8 years after a primary TKA. The primary complaint of the patient

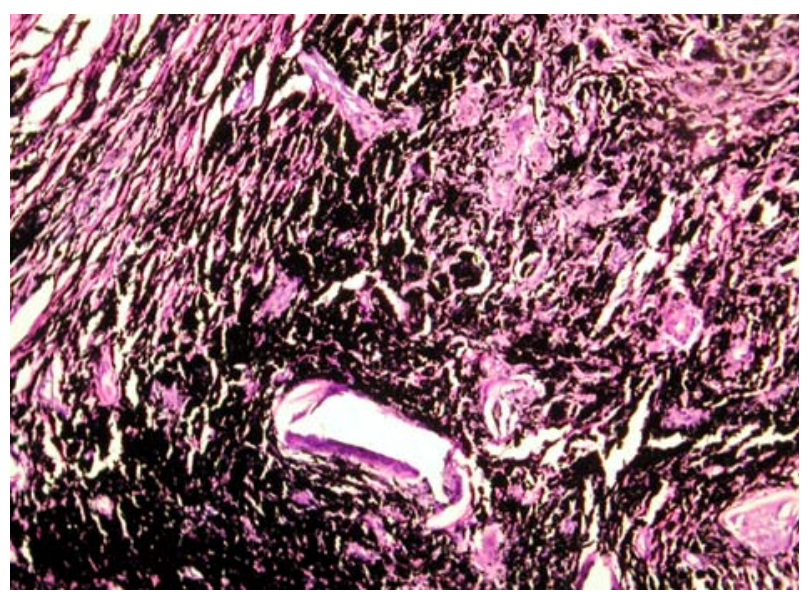

Fig. 4 Polarized light microscopic examination shows a foreign body reaction with giant cells around crystals of polyethylene debris

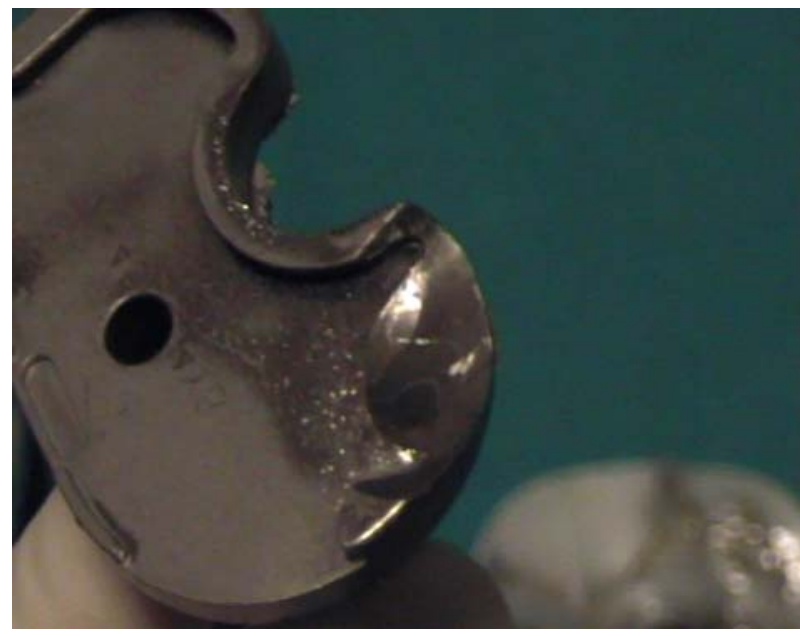

Fig. 5 Photograph showing retrieved tibial component showing an import usure on the medial side

was pain associated with other symptoms close to those of a trombophlebitis. Instrumental investigations ruled out deep venous thrombosis.

A dissecting popliteal cyst in a failed TKA was diagnosed. A two-stage surgical procedure, consisting in cyst excision at first and secondly a revision TKA, was carried out.

Dissecting popliteal cyst [3] after the TKA is a rare event reported in the literature $[4,5,7,11,12]$. Pavlov [12] reported about two cases of dissecting popliteal cysts after the TKA. The patients complained of symptoms close to those of vein thrombosis, with no signs of implant loosening; repeated aspirations of cyst were carried out with good results. Dirschl [5] described about three patients who underwent a revision of TKA without excision of the popliteal cyst. Hsu [7] described one case caused by a dislocated metallic

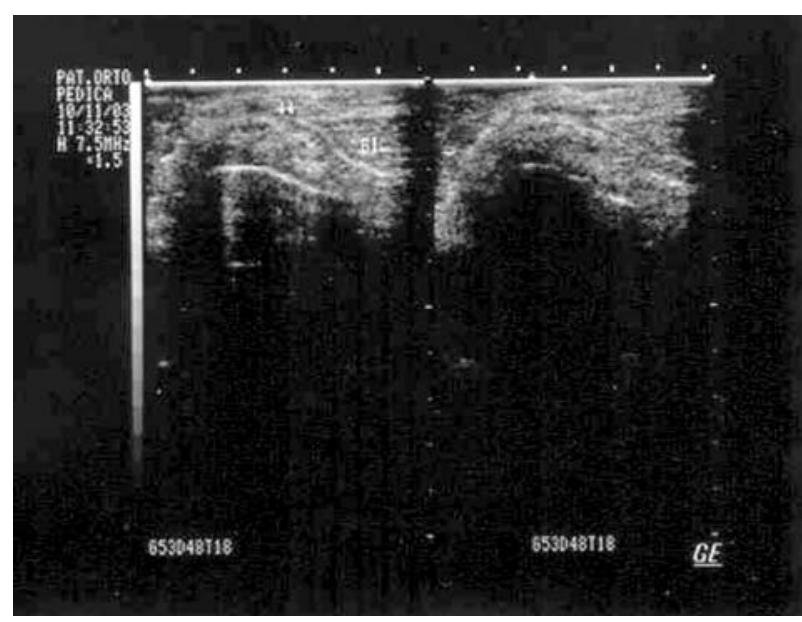

Fig. 6 Postoperative ultrasound investigation ruled out a recurrence of popliteal cyst 
part of the patellar component cutting the posterior knee capsule, and created a passage into the posterolateral popliteal fossa. A staged operation consisting of revision of the TKA, followed by a complete excision of the popliteal cyst was the surgical option. Niki [11] reported one case of gigantic popliteal synovial cyst 8 years after the TKA. The patient complained of pain and swelling of the knee without radiological signs of implant loosening. During operation, damage to the polyethylene insert was observed, associated to the proliferation of villous synovial tissue. Synoviectomy as well as excision of the synovial cyst and replacement of the polyethylene insert was performed. Chan [4] was the first author to report a two-stage procedure for the treatment of a large dissecting popliteal cyst after failed TKA, in which the main complaint was pain in the posterior region of the knee. The rationale behind this surgical management was to first relieve the pain. Like Chan [4], we chose to perform a two-stage procedure because the severe pain related to the cyst was the main complaint of the patient. Our opinion was that the large cyst could complicate the revision procedure, therefore if removed first, not only the pain would diminish, but we could also prevent the cyst recurrence, and take out the polyethylene debris [4]. The cyst was filled with fluid containing polyethylene debris, indicating a worn out TKA $[4,5]$. We performed the second stage of the procedure 5 months later because of health problems related to the patient's medical condition, limiting an earlier operation. When this option of a two-stage revision is considered, an interval of $4-6$ weeks is sufficient, as described by Chang [4].

The literature outlines the importance of flow cytometric analysis before surgery, in order to detect polyethylene debris phagocytosed by macrophages [11]. The increased proportion of the macrophages with particles indicates a particle-induced synovitis and synovial cyst formation. This finding is a sign of a connection between the joint cavity and the cyst, and it might be considered as a "preceding sign" of osteolysis, wear out of implanted components, as well as loosening of the prosthesis. In our case, this analysis was not carried out because of radiological evidence of TKA loosening. This point is really important; in fact, although one may suggest that a better follow-up for a TKA, including X-rays studied at 1, 2, 5, 10 years could avoid the stage of the polyethylene (and other components) wear, or at least lead to an earlier diagnosis, preventing the formation of a popliteal cyst. However, Niki [11] has described one case of popliteal cyst after TKA without radiological signs of implant loosening and surgical findings of a damaged polyethylene insert.

Various studies focusing on the biology of periprosthetic osteolysis, as well as prosthesis loosening revealed that wear debris including particles of polyethylene, different metals and polymethyl-metacrylate originating from prosthesis itself cause particle-induced synovitis, fibroblastic proliferation, macrophage phagocitosis, tumor necrosis factor- $\alpha$ secretion and foreign particle reaction $[6,8,10,11,13]$. The high intraarticular pressure, caused by particle-induced joint effusion, exceeds the saturation level of synovial uptake, and that way contributes to cyst formation [2]. We recognized two patterns of cysts: cystic bone lesions communicating with the bone-implant interface, and synovial cysts in the bursa, or other soft tissue [11]. This latter pattern is not always associated with prosthesis loosening, but the investigations should rule it out $[11,12]$.

In conclusion, a dissecting popliteal cyst in presence of a TKA might be a sign of wear particles generation. The cyst contains polyethylene debris, and should be excised in order to eliminate the polyethylene debris that is the inducing factor for prosthetic loosening. The surgical management depends on the patient's symptoms. A two-stage procedure, consisting of excision of the cyst first, followed after an adequate period of time by a revision TKA, may be a useful alternative, especially in clinical circumstances, such as with old patients associated with other medical conditions; for those patients, a two-stage procedure with a short time interval may be difficult to perform

\section{References}

1. Adams R (1840) Chronic rheumatoid arthritis of the knee. J Med Sci 17:520

2. Anthony PP, Gie GA, Howie CR et al (1990) Localized endosteal bone lysis in relation to the femoral components of cemented total hip arthroplasties. J Bone Joint Surg Br 72:971-976

3. Burleson RJ, Bickel WH, Dahlin DC (1956) Popliteal cyst: a clinicopathology survey. J Bone Joint Surg A 38:1265-1270

4. Chan Yi-Seng, Wang Ching-Jen, Shin Chun-Hsiung (2000) Two stage operation for treatment of a large dissecting popliteal cyst after failed TKA. J Arthroplasty 15:1068-1072

5. Dirschl DR, Lachiewicz PF (1992) Dissecting popliteal cyst as the presenting symptom of a malfunctioning TKA. Report of four cases. J Arthroplasty 7:37-41

6. Gross TP, Lennox DW (1992) Osteolytic cyst-like area associated with polyethylene and metallic debris after total knee replacement with an uncemented vitallium prosthesis:a case report. J Bone Joint Surg A 74:1096-1101

7. Hsu Wei-Hsiu, Hsu Wen-Wei Robert, Huang TsunJen, Lee Kam-Fui (2002) Dissecting popliteal cyst resulting from fragmented, dislodged metal part of the patellar component after total knee arthroplasty. J Arthroplasty 17:792-797 
8. Jacobs JJ, Rosenbaum DH, Hay RM, Gitelis S, Black J (1992) Early sarcomatous degeneration near a cementless hip replacement: a case report and review. J Bone Joint Surg Br 74:740-744

9. Katz RS, Zizic TM, Arnold WP (1977) The pseudothrombophebitis syndrome. Medicine (Baltimore) 56:151

10. Mari M (1999) Radiographic evaluation of the painful total knee replacement. In: Lotke PA, Garino JP (eds) Revision total knee arthroplasty. Lippincot-Raven, New York, p 115
11. Niki Y, Matsumoto H, Otani T, Yoshimine F, Inokuchi W, Morisue H (2003) Gigantic popliteal synovial cyst caused by wear particles after TKA. J Arthroplasty 18:1071-1075

12. Pavlov H, Steinbach L, Fried SH (1983) A posterior ascending popliteal cyst mimicking thrombophebitis following TKA. Clin Orthop 179:204-208

13. Visuri T, Pukkala E, Paavolainen P, Pulkkinen P, Riska EB (1996) Cancer risk after metal on metal and polyethylene on metal total hip arthroplasty. Clin Orthop 329S:S280-S289 